\title{
IL-10 Plays a Pivotal Role in Tamoxifen-Induced Spasmolytic Polypeptide-Expressing Metaplasia in Gastric Mucosa
}

\author{
Chansu Lee ${ }^{1}$, Hyuk Lee ${ }^{2}$, Seo Yun Hwang ${ }^{1}$, Chang Mo Moon ${ }^{3}$, and Sung Noh Hong ${ }^{2}$ \\ ${ }^{1}$ Samsung Biomedical Research Institute, Samsung Medical Center, ${ }^{2}$ Department of Medicine, Samsung Medical Center, Sungkyunkwan \\ University School of Medicine, and ${ }^{3}$ Department of Internal Medicine, Ewha Womans University School of Medicine, Seoul, Korea
}

\section{See editorial on page 741 .}

Background/Aims: Gastric cancer evolves in the pathologic mucosal milieu, and its development is characterized by both the loss of acid-secreting parietal cells and mucosal cell metaplasia, called spasmolytic polypeptide-expressing metaplasia (SPEM). Cytokines, such as interleukin (IL)-10, IL-1 $\beta$, and IL-6, play a key role in gastric carcinogenesis. However, changes in the cytokine profile of SPEM have not been evaluated. Methods: To induce SPEM in mouse stomachs, C57BL/6 mice were intraperitoneally injected with tamoxifen and sacrificed at 3,10 , and 21 days after treatment. RNAsequencing (RNA-seq) and a multiplex bead array were used to measure cytokines in the stomachs of tamoxifen-treated/ control mice. Results: The administration of tamoxifen led to the rapid development and histological normalization of SPEM 3 and 10 days after administration, respectively. RNAseq revealed that the expression of IL-10 was decreased 3 days after tamoxifen administration. The multiplex assay identified a significant decline in IL-10 levels 3 days after tamoxifen treatment $(58.38 \pm 34.44 \mathrm{pg} / \mathrm{mL}$ vs $94.09 \pm 4.98$ $\mathrm{pg} / \mathrm{mL}, \mathrm{p}=0.031$ ), which normalized at 10 and 21 days after tamoxifen treatment. Immunofluorescence staining confirmed that IL-10 expression was markedly decreased at the time of SPEM development and subsequently returned to normal, accompanied by a reversal in histologic changes. Conclusions: IL-10 may play a pivotal role in the tamoxifeninduced acute development of gastric SPEM. (Gut Liver

2017;11:789-797)
Key Words: Stomach neoplasms; Spasmolytic polypeptideexpressing metaplasia; Interleukin-10

\section{INTRODUCTION}

In the stomach, to initiate digestion and protect the epithelial structure, the oxyntic mucosa secretes and regulates various molecules, such as acids, zymogens, histamines, somatostatin, and other local hormones. ${ }^{1}$ The oxyntic mucosa is composed a set of secretory cells, including parietal cells (PCs), chief cells, mucosal neck cells, and several types of endocrine cells. ${ }^{2-4}$ Among them, PCs appear to be critical regulators of cellular homeostasis in the gastric gland and numerous studies have revealed that PC loss or dysfunction result in decreased numbers of zymogenic cells. ${ }^{3,5}$ Zymogenic cell loss is accompanied by expansion of a dysplastic cell type, i.e., spasmolytic polypeptideexpressing metaplasia (SPEM), which shares features with cells of the mucosal neck-zymogenic lineage.

Recent studies suggest that SPEM can initiate gastric carcinogenesis, and it is considered a reversible early precursor of cancerous lesions. ${ }^{3-7}$ The cytokines produced by mucosal cells, such as interleukin (IL)-4, vascular endothelial growth factor (VEGF), interferon (IFN)- $\gamma$, tumor necrosis factor (TNF)- $\alpha$, and IL-10, are related to metaplasia in several tissues, including the stomach. $^{8-11}$ Accordingly, the aberrant expression of cytokines in gastric oxyntic epithelial cells is associated with the development of metaplasia, ${ }^{12,13}$ but alterations in the expression patterns of cytokines have not been evaluated in cases of SPEM, an early stage of metaplasia during gastric carcinogenesis.

Correspondence to: Sung Noh Hong and Chang Mo Moon ${ }^{\mathrm{b}}$

${ }^{a}$ Department of Medicine, Samsung Medical Center, Sungkyunkwan University School of Medicine, 81 Irwon-ro, Gangnam-gu, Seoul 06351, Korea Tel: +82-2-3410-3409, Fax: +82-2-3410-6983, E-mail: gisnhong@gmail.com

${ }^{b}$ Department of Internal Medicine, Ewha Womans University Mokdong Hospital, Ewha Womans University School of Medicine, 1071 Anyangcheon-ro, Yangcheon-gu, Seoul 07985, Korea

Tel: +82-2-2650-2945, Fax: +82-2-2655-2076, E-mail: drmcm75@naver.com

Received on September 8, 2016. Revised on November 11, 2016. Accepted on March 7, 2017. Published online June 26, 2017

pISSN 1976-2283 eISSN 2005-1212 https://doi.org/10.5009/gnl16454

Chansu Lee and Hyuk Lee contributed equally to this work as first authors.

@) This is an Open Access article distributed under the terms of the Creative Commons Attribution Non-Commercial License (http://creativecommons.org/licenses/by-nc/4.0) which permits unrestricted non-commercial use, distribution, and reproduction in any medium, provided the original work is properly cited. 
SPEM can be induced rapidly, as demonstrated when mice are infected with Helicobacter species or treated with DMP 777 or tamoxifen. ${ }^{14-17}$ Tamoxifen, a selective estrogen receptor modulator, most rapidly induces PC loss and SPEM in mice. In addition, the metaplastic mucosa of tamoxifen-treated mice tends to recover in a time-dependent manner. ${ }^{17}$ In this study, we investigated the expression change in cytokine profiles during the progression and recovery of SPEM using mice in which SPEM was induced by tamoxifen.

\section{MATERIALS AND METHODS}

\section{Animal experiments}

Eight-week-old C57BL/6 mice were purchased from the Jackson Laboratory (Bar Harbor, ME, USA). Tamoxifen (3 mg/20 g mouse body weight; Cayman Chemical, Ann Arbor, MI, USA) or the same volume of vehicle was injected intraperitoneally once daily for 3 consecutive days to induce SPEM. Tamoxifen was dissolved in 10\% ethanol and 90\% sunflower seed oil (Sigma, St. Louis, MO, USA). Groups of six mice each were sacrificed at 3, 10, and 21 days after tamoxifen or vehicle administration.

\section{Histological analyses}

Stomach tissue obtained from mice was fixed in neutral buffered 10\% formalin for 12 to 24 hours, washed in $70 \%$ ethanol, processed by standard methods, embedded in paraffin, sectioned at $3 \mu \mathrm{m}$, and stained with hematoxylin and eosin (H\&E). Loss of PCs and changes in the differentiation of mucosal neck-zymogenic lineage cells were observed. PCs were counted in H\&Estained sections taken from every mouse used in the study. Fifty well-aligned corpus gastric units were selected at random from each mouse. The number of PCs counted in each unit and the average number of PCs/unit were calculated. For immunofluorescence staining, stomach sections were co-stained with lectin GS II (a mucosal neck cell marker, 1:100; Thermo Fisher Scientific, Waltham, MA, USA) and anti-VEGF- $\beta$ (PCs marker, 1:200; Santa Cruz Biotechnology, Dellas, TX, USA) antibodies. H\&E counts were indistinguishable from immunofluorescence-based counts.

\section{Total RNA extraction and RNA sequencing}

Total RNA was isolated from fresh frozen tissues of mouse stomach sacrificed 3 days after tamoxifen or vehicle administration (n=3/group) using an RNeasy Mini Kit (Qiagen, Valencia, CA, USA) according to the manufacturer's instructions. Total RNA quality and quantity were determined using a Nanodrop 8000 UV-Vis spectrometer (Thermo Fisher Scientific) and an Agilent 2100 Bioanalyzer (Agilent Technologies, Santa Clara, CA, USA). The quantity of all RNA samples was $>2 \mu \mathrm{g}$, and the quality had an RNA integrity number value >8.0.

Library construction for whole transcriptome sequencing was performed using the TruSeq RNA sample preparation v2 kit
(Illumina, San Diego, CA, USA). Isolated total RNA ( $2 \mu \mathrm{g})$ was used for reverse transcription reactions with poly (dT) primers and SuperScript ${ }^{\mathrm{TM}}$ II reverse transcriptase (Invitrogen/Life Technologies, Grand Island, NY, USA) according to the manufacturer's protocols. Briefly, the RNA sequencing (RNA-seq) library was prepared through cDNA amplification, end-repair, 3' end adenylation, adapter ligation, and amplification. The quality and concentration of the library were measured using Bioanalyzer and Qubit instruments, respectively. Sequencing of the transcriptome library was carried out using the $100 \mathrm{bp}$ paired-end mode of the TruSeq Rapid PE Cluster kit and the TruSeq Rapid SBS kit (Illumina).

\section{RNA-seq data analysis}

Reads from FASTQ format files were mapped against the mouse reference genome (UCSC mm10) using TopHat version 2.0.6 with default parameters (http://ccb.jhu.edu/software/ tophat/index.shtml). The mapping results were analyzed by HTSeq version 0.6.1 to quantify transcript abundance. Coding genes were selected and raw read counts were normalized by TMM (trimmed mean of M-values) normalization. We analyzed the expression levels of cytokines and their receptors using the DESeq $R$ package based on fold change.

\section{Reverse transcription polymerase chain reaction}

Total RNA was isolated from fresh frozen tissues of mouse stomach sacrificed before and after vehicle (3 days) or tamoxifen administration (3, 10, and 21 days). Reverse transcription polymerase chain reaction (RT-PCR) was performed with the following primers: IL-10 (forward: 5'-TAAGGCTGGCCACACTTGAG-3', reverse: 5'-GTTTTCAGGGATGAAGCGGC-3'); IL-12p40 (forward: 5'-AGGTCACACTGGACCAAAGG-3', reverse: 5'-TGGTTTGATGATGTCCCTGA-3'); IL-12p35 (forward: 5'-CATCGATGAGCTGATGCAGT-3', reverse: 5'-CAGATAGCCCATCACCCTGT-3'); IL-1 $\beta$ (forward: 5'-TGCCACCTTTTGACAGTGATG-3', reverse: 5'-TTCTTGTGACCCTGAGCGAC-3'); IL-5 (forward: 5'-ATGGAGATTCCCATGAGCAC-3', reverse: 5'-CCCACGGACAGTTTGATTCT-3'); TNF- $\alpha$ (forward: $5^{\prime}$-TGCCAGCTCCAGGATTTCAG-3', reverse: 5'-CTCAGCCCTCACTTGACCTG-3'); INF- $\gamma$ (forward: 5'-TCCCCAAGAACATGCTCTCTAA-3', reverse: 5'-TTCCTCGAACTCCACACTGC-3'); and GAPDH (forward: 5'-ACCCAGTCCATGCCATCAC-3', reverse: 5'-TCCACCACCCTGTTGCTGTA-3').

\section{Multiplex immunoassay}

Stomach tissues obtained from mice were immediately frozen in liquid nitrogen and stored at $-70^{\circ} \mathrm{C}$. Cytokine levels were determined in tissue lysates using a multiplex mouse bead immunoassay kit (Bio-Rad, Hercules, CA, USA). All samples were lysed with T-PER tissue protein extraction reagent (Thermo Fisher Scientific). The nine-plexed assays (INF- $\gamma$, IL-12p70, IL4 , IL-5, IL-6, TNF- $\alpha$, IL-10, IL17A, and IL-1 $\beta$ ) were performed in 
accordance with the manufacturer's instructions. Optical density was determined using a microplate reader set to $450 \mathrm{~nm}$.

\section{Immunofluorescence}

Stomach tissues obtained from mice were prepared, stained, and imaged to assess cytokine expression. The primary antibodies used for immunofluorescence staining were: lectin GS II, anti-VEGF- $\beta$, anti-IL-10 (1:200; Santa Cruz Biotechnology), anti-IFN- $\gamma$ (1:50; Abcam, Cambridge, UK), anti-IL-6 (1:500; Abcam), anti-TNF- $\alpha$ (1:100; Abcam), anti-IL-1 $\beta$ (1:100; Abcam), and anti-proteinase 3 (Santa Cruz Biotechnology). Expression levels of VEGF- $\beta$, GS II, and cytokines were evaluated under confocal microscopy after immunofluorescent staining of deparaffinized tissue sections.

\section{Statistics}

Continuous variables are expressed as means \pm standard devia- tion, while categorical variables are presented as absolute values and percentages. Differences between continuous variables were analyzed using unpaired Student t-tests, while differences between categorical variables were analyzed using the chi-square test and Fisher exact test. All statistical analyses were performed with SPSS software version 18.0 (SPSS Inc., Chicago, IL, USA).

\section{RESULTS}

\section{Tamoxifen induces loss of PCs in the gastric mucosa of mice}

We confirmed that treatment of normal mice with three consecutive administrations of $3 \mathrm{mg} / 20 \mathrm{~g}$ body weight of tamoxifen leads to apoptosis of about 90\% of all gastric PCs and metaplasia of mucosal neck-zymogenic lineage cells within 3 days. Gastric histology returns to nearly normal by 10 days. As shown in Fig. 1A, significant PC loss was detected in tamoxifen-

A

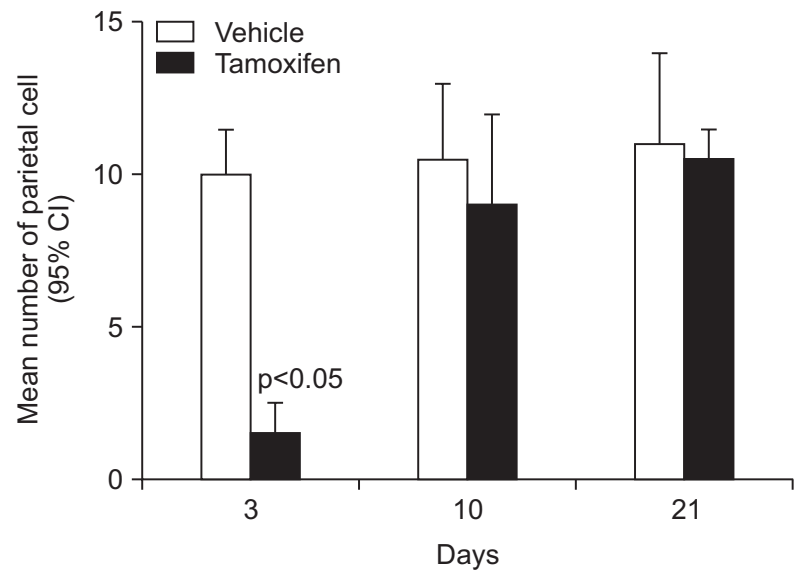

B

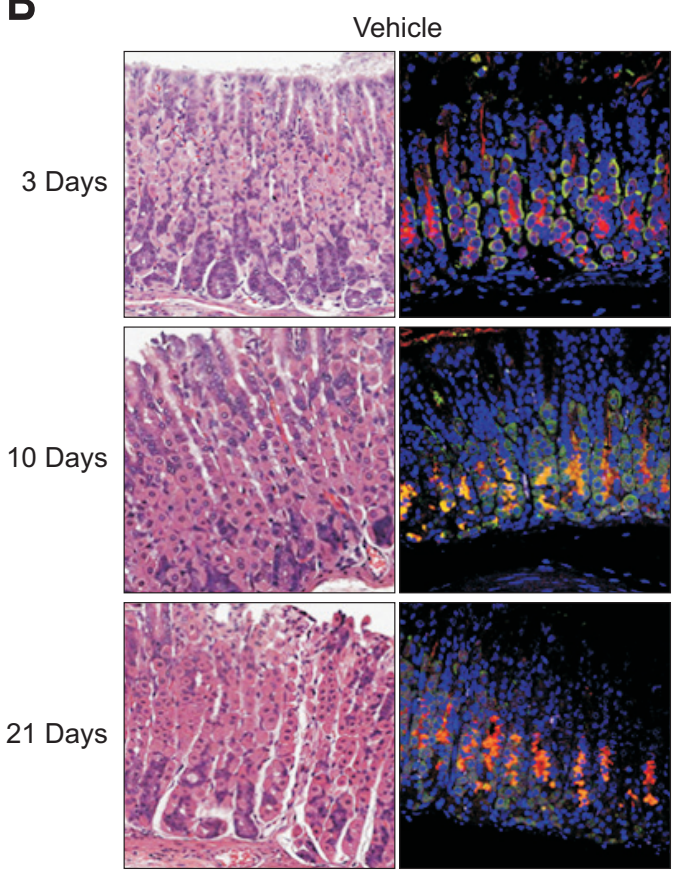

Tamoxifen
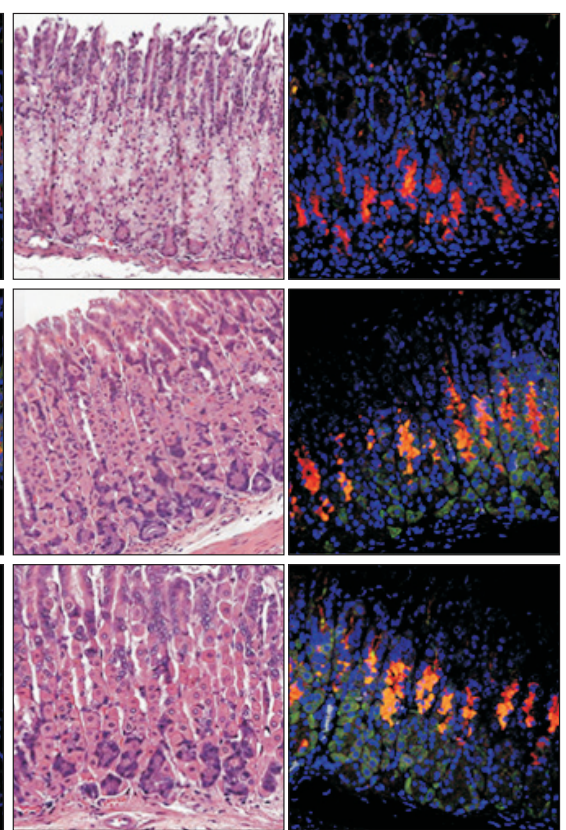

Fig. 1. Tamoxifen induces a decrease in the number of parietal cells (PCs) in the gastric mucosa of mice. (A) Mice were treated with 3 $\mathrm{mg} / 20 \mathrm{~g}$ body weight of tamoxifen or vehicle for 3 days. Experimental mice were sacrificed at the indicated time points after the final tamoxifen administration. PCs were counted in hematoxylin and eosin-stained sections. (B) The expression levels of vascular endothelial growth factor (VEGF)- $\beta$ and GS II were evaluated by confocal microscopy after immunofluorescence staining of deparaffinized tissue sections (red: GS II, a mucosal neck cell marker; green: VEGF- $\beta$, a PC marker). $\mathrm{CI}$, confidence interval. 
treated mice at 3 days when compared to mice in the control group $(\mathrm{p}<0.05)$. These differences were not detected at 10 or 21 days, suggesting that PCs recovered. To confirm these findings, we performed immunofluorescence staining of the gastric gland.
As shown in Fig. 1B, anti-VEGF- $\beta$, a PC marker, was not detected at 3 days; however, PCs had recovered at 10 and 21 days. None of the mucus neck cells changed at any point in time, suggesting that tamoxifen induces rapid and reversible SPEM

Table 1. The Expression Pattern of Cytokines and Their Receptors in the Mouse Stomach 3 Days after Vehicle or Tamoxifen Administration

\begin{tabular}{|c|c|c|c|c|}
\hline & Gene symbol & Base mean of control* & Base mean of case ${ }^{\dagger}$ & Fold change \\
\hline \multicolumn{5}{|l|}{ Proinflammatory } \\
\hline $\mathrm{IL}-1 \alpha$ & IL1A & 12.5 & 24.0 & 1.92 \\
\hline $\mathrm{IL}-1 \beta$ & IL1B & 12.0 & 6.0 & 0.50 \\
\hline IL-1 receptor & IL1R1 & 563.5 & 626.0 & 1.11 \\
\hline IL-6 & IL6 & 0.0 & 1.0 & NA \\
\hline IL-6 receptor & IL6RA & 205.0 & 244.5 & 1.19 \\
\hline IL-12, subunit p35 & IL12A & 0.0 & 0.0 & NA \\
\hline IL-12, subunit p40 & IL12B & 0.0 & 0.0 & NA \\
\hline IL-12 receptor, subunit $\beta 1$ & IL12RB1 & 2.0 & 0.5 & 0.25 \\
\hline IL-12 receptor, subunit $\beta 2$ & IL12RB2 & 24.0 & 22.5 & 0.94 \\
\hline TNF- $\alpha$ & TNF & 1.0 & 0.5 & 0.50 \\
\hline TNF receptor & TNFRSF1A & $1,965.5$ & $2,068.0$ & 1.05 \\
\hline IFN- $\gamma$ & INFG & 0.0 & 0.0 & NA \\
\hline IFN- $\gamma$ receptor & IFNGR1 & $3,487.5$ & $3,808.5$ & 1.09 \\
\hline $\mathrm{IL}-17$ & IL17A & 0.0 & 0.0 & NA \\
\hline IL-17 receptor A & IL17RA & 315.0 & 384.5 & 1.22 \\
\hline IL-17 receptor C & IL17RC & $1,075.5$ & $1,309.5$ & 1.22 \\
\hline $\mathrm{IL}-21$ & IL21 & 0.0 & 0.0 & NA \\
\hline IL-21 receptor & IL21R & 4.5 & 5.0 & 1.11 \\
\hline $\mathrm{IL}-22$ & IL22 & 0.0 & 0.0 & NA \\
\hline IL-22 receptor & IL22RA1 & 295.0 & 333.5 & 1.13 \\
\hline \multicolumn{5}{|l|}{ Anti-inflammatory or immune-regulatory } \\
\hline $\mathrm{IL}-4$ & IL4 & 0.5 & 0.5 & 1.00 \\
\hline IL-4 receptor & IL4RA & 664.0 & 872.5 & 1.31 \\
\hline IL-5 & IL5 & 0.68 & 0.31 & 0.46 \\
\hline IL-5 receptor, subunit $\alpha$ & IL5RA & 0.0 & 0.5 & NA \\
\hline GM-CSF/IL-3/IL-5 receptor common $\beta$ subunit & Csf2rb & 54.5 & 42.0 & 0.77 \\
\hline $\mathrm{IL}-13$ & IL13 & 0.0 & 0.0 & NA \\
\hline IL-13 receptor, $\alpha 1$ & IL13RA1 & $1,705.0$ & $2,125.0$ & 1.25 \\
\hline $\mathrm{IL}-10$ & IL10 & 2.0 & 0.5 & 0.25 \\
\hline IL-10 receptor, subunit $\alpha$ & IL10RA & 41.0 & 41.5 & 1.01 \\
\hline IL-10 receptor, subunit $\beta$ & IL10RB & $1,224.5$ & $1,348.0$ & 1.10 \\
\hline TGF- $\beta 1$ & TGFB1 & 173.5 & 188.5 & 1.09 \\
\hline TGF- $\beta 2$ & TGFB2 & 106.5 & 101.5 & 0.95 \\
\hline TGF- $\beta 3$ & TGFB3 & 139.0 & 114.0 & 0.82 \\
\hline TGF- $\beta$ receptor 1 & TGFBR1 & 931.0 & 937.0 & 1.01 \\
\hline TGF- $\beta$ receptor 2 & TGFBR2 & $2,059.5$ & $2,063.5$ & 1.00 \\
\hline TGF- $\beta$ receptor 3 & TGFBR3 & 630.0 & 756.0 & 1.20 \\
\hline
\end{tabular}

IL, interleukin; NA, not available; TNF, tumor necrosis factor; IFN, interferon; GM-CSF, granulocyte macrophage colony-stimulating factor; TGF, transforming growth factor.

*Base mean of mouse stomachs sacrificed 3 days after vehicle administration; ${ }^{\dagger}$ Base mean of mouse stomachs sacrificed 3 days after tamoxifen administration. 
in C57BL/6 mice. There were no significant changes in inflammatory cell infiltration in the lamina propria or submucosa at 3 , 10 , or 21 days after tamoxifen or vehicle administration.

\section{Expression pattern of cytokines and their receptors assessed using RNA-seq}

RNA-seq was performed using two pairs of fresh frozen samples of mouse stomach. Mice were sacrificed 3 days after intraperitoneal tamoxifen or vehicle administration. We focused on the expression pattern of cytokines and their receptors. Although most cytokine and receptor transcripts showed very low expression levels, the fold change of expression of IL-1 $\beta$, IL-12

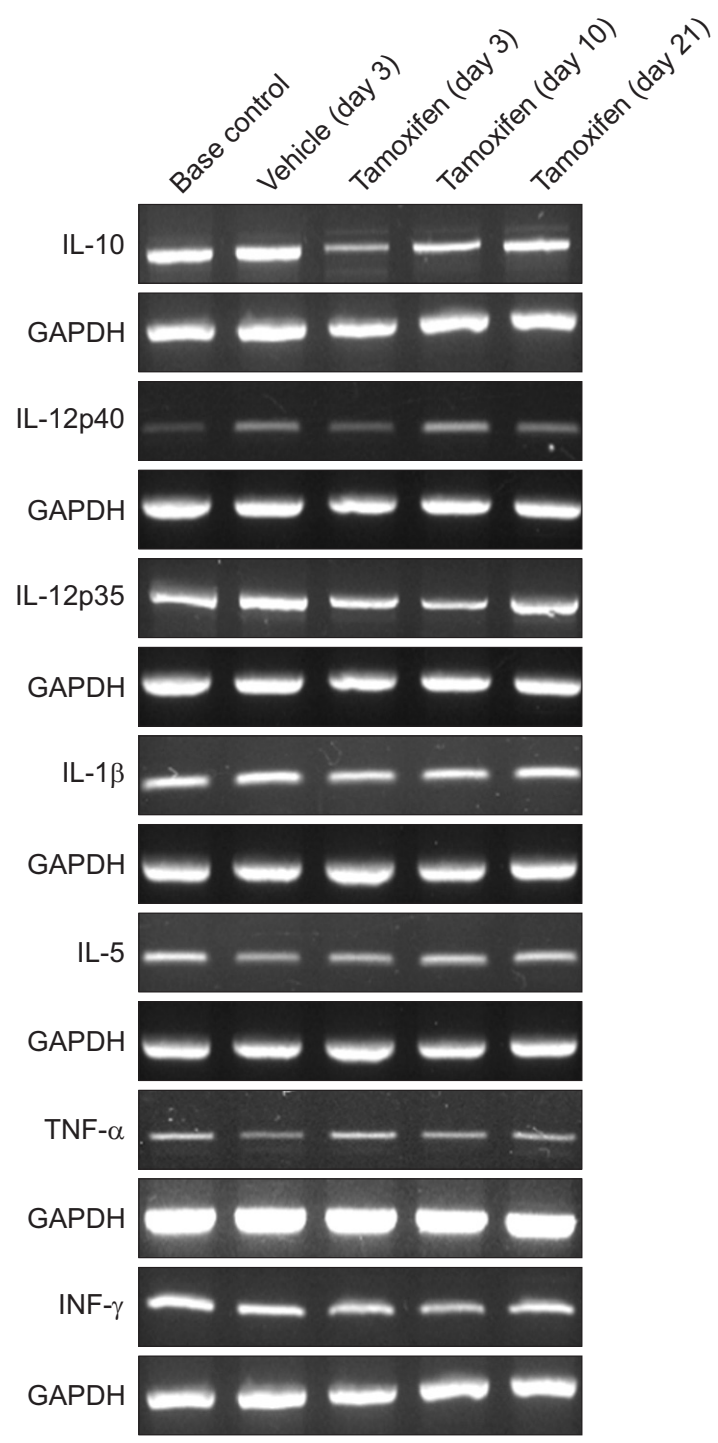

Fig. 2. The reverse transcription polymerase chain reaction results showing the cytokine profiles in tamoxifen-induced reversible gastric metaplasia. The transcripts of interleukin (IL)-10, IL-12p40, IL$12 \mathrm{p} 35$, IL-1 $\beta$, IL-5, tumor necrosis factor (TNF)- $\alpha$, and interferon (IFN)- $\gamma$ were analyzed in the stomach tissues of mice sacrificed before and after vehicle (3 days) or tamoxifen administration (3, 10, and 21 days). All experiments were independently repeated three times. GAPDH, glyceraldehyde 3-phosphate dehydrogenase. receptor subunit $\beta 1, \mathrm{TNF}-\alpha$, IL-5, and IL-10 in mouse stomach at 3 days after tamoxifen administration were $\geq 2$ or $\leq 0.5$ (Table 1).

RT-PCR confirmed that the expression of IL-10 was lower in mouse stomach at 3 days after tamoxifen administration than in mouse stomach before administration or following vehicle administration. The expression of IL-10 recovered at 10 and 21 days after tamoxifen administration (Fig. 2).

\section{Cytokine expression in multiplex immunoassay and immunofluorescence staining}

Since the expression of cytokines was altered by SPEM progression, we performed a multiplex immunoassay to confirm changes in expression levels of inflammatory cytokines in samples from tamoxifen-treated mice. Protein levels of IL-1 $\beta$, IL12 p70, TNF- $\alpha$, IL-5, IL-10, IFN- $\gamma$, IL-4, and IL- 6 were examined in mouse gastric tissues. We analyzed the change in expression patterns according to PC loss and subsequent restoration. Cytokine levels in the gastric tissues of tamoxifen-treated mice were not statistically different from those in control mice, although the levels of cytokine expression, except IL-10, showed constitutive increasing patterns in tamoxifen-treated mouse tissues (Fig. 3). IL-10 was significantly reduced after 3 days ( $p=0.031$ ), and subsequently recovered at days 10 and 21, which was accompanied by a reverse in histologic changes.

IL-10 was not detected at 3 days but had recovered at 10 and 21 days. This suggests that the dysregulation of epithelial IL10 may be associated with tamoxifen-induced SPEM in gastric mucosa. To define the relationship between IL-10 expression and PC loss/recovery, we co-stained gastric glands with antiIL-10 and anti-VEGF- $\beta$ antibodies. As shown in Fig. 4A, IL10 was detected in the cytoplasm, where it co-localized with VEGF- $\beta$. This suggests that IL-10 expression may be regulated by PC reduction and that IL-10 expression was associated with tamoxifen-induced SPEM.

Given that cytokines can be an important factor in the progression of mucosal metaplasias, such as SPEM, we directly examined the expression levels of cytokines in tamoxifen-treated mouse tissues using immunofluorescence staining for IFN- $\gamma$, IL-6, TNF- $\alpha$, and IL-1 $\beta$, which are associated with gastric carcinogenesis. ${ }^{8-11}$ Because tamoxifen-induced SPEM is not related to inflammation or inflammatory cell infiltration, we evaluated cytokine changes in epithelial cells during SPEM by immunofluorescence staining. However, there were no changes in the expression levels of IFN- $\gamma$, IL-6, TNF- $\alpha$, or IL- $1 \beta$ during SPEM, which suggests that they have no relation to tamoxifen-induced SPEM (Fig. 4B).

\section{DISCUSSION}

Tamoxifen is used a chemotherapeutic drug to treat diverse tumors. However, some reports have suggested that tamoxifen increases the risk of endometrial cancer and subsequent gastric 

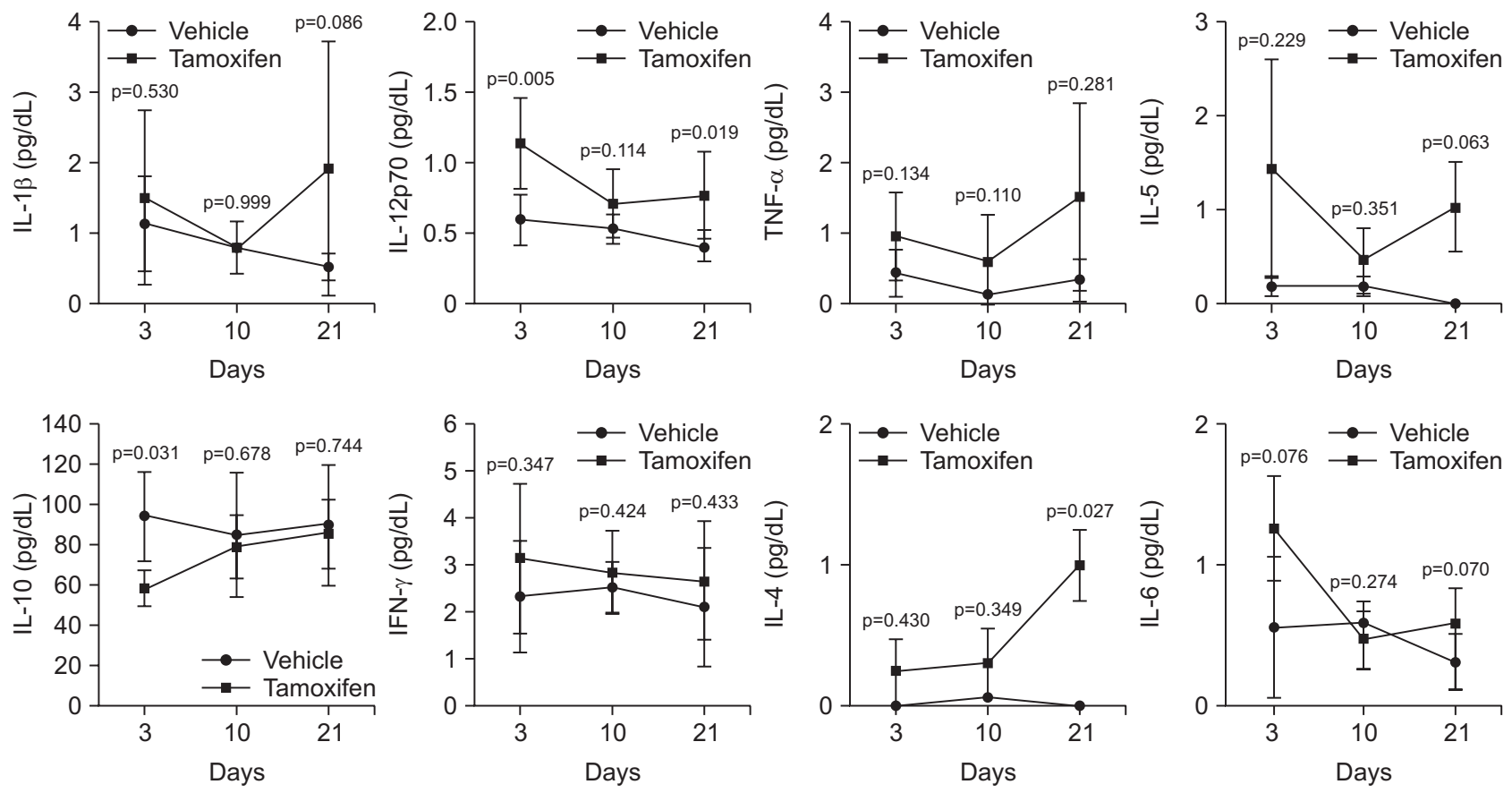

Fig. 3. A multiplex immunoassay measuring the levels of cytokines in tamoxifen-treated mouse stomachs. Whole gastric tissue lysates were extracted from tamoxifen-induced spasmolytic polypeptide-expressing metaplasia mice and control mice. The experimental mice were sacrificed at the indicated time points after tamoxifen administration. Cytokine levels were measured in the tissue lysates using a multiplex mouse bead immunoassay kit. The optical density was determined using a microplate reader set to $450 \mathrm{~nm}$. The expression levels of interleukin (IL)-1 $\beta$, IL-12p70, tumor necrosis factor (TNF)- $\alpha$, IL-5, IL-10, interferon (IFN)- $\gamma$, IL-4, and IL-6 were analyzed. The data shown are the means \pm standard error of the mean of three independent experiments.

cancer. ${ }^{18,19}$ Huh et al. ${ }^{17}$ showed that tamoxifen treatment induced gastric atrophy and SPEM in both male and female mice, and its effects were dependent on dose and time. In this study, we administrated tamoxifen to C57BL/6 mice and observed that the number of PCs decreased in tamoxifen-treated gastric glands after 3 days and recovered after 10 days. PCs have important roles in regulating cellular growth and cell lineage differentiation in gastric mucosa. Loss of PCs is induced by oxyntic atrophy, which results in SPEM. ${ }^{4}$ These results suggest that tamoxifen induces rapid and reversible SPEM in C57BL/6 mice.

A number of cytokines are known to regulate immune response and homeostasis in the gastric mucosa. ${ }^{20}$ Recent reports also showed that the expression of pro- and anti-inflammatory cytokines is related to the development of atrophic gastritis and mucosal cell metaplasia. ${ }^{12,13}$ Thus, altered cytokine expression could be related to the initiation of SPEM. In the results of the RNA-seq, cytokines and receptor transcripts, including IL-10, IL-1 $\beta$, IL-5, and TNF- $\alpha$, in mouse stomach 3 days after tamoxifen administration showed half or 2-fold higher level of those in controls. However, in RT-PCR, it seemed that the expression of IL-10 only were reduced, and the levels of IL-1 $\beta$, IL-5, and TNF- $\alpha$ were unchanged. There was a discrepancy between the results of RNA-seq and RT-PCR. RNA-seq provides a broad view of genes, which is suitable for investigating the effect of large numbers of genes, detecting alternative splicing, and making small RNA characterizations. However, the data obtained from
RNA-seq are broad and relative, and the sensitivity of the data is based on the read depth or the length of the targeted genome. RT-PCR is limited to studying a few genes at a time; however, it can be utilized to quantify a set of genes that might be sensitive. The results suggest that these pro- and anti-inflammatory cytokines may play roles in the progression of SPEM. The expression pattern of IL-10 indicates that tamoxifen-induced SPEM develops regardless of inflammation and IL-10 has a significant role in the progression of SPEM.

IL-10 is a multifunctional anti-inflammatory cytokine that downregulates cell-mediated immune responses and cytotoxic inflammatory responses. Changes in IL-10 levels have been observed during the initiation and progression of gastric cancer. ${ }^{21}$ In chronic gastric inflammation, activated neutrophils and mononuclear cells produce different types of cytokines that are crucial in regulating inflammation In chronic gastric inflammation, activated neutrophils and mononuclear cells produce different types of cytokines that are crucial for regulating inflammation. IL-10 is secreted by gastric mucosal tissues and may protect the mucosa by limiting tissue damage caused by inflammation secondary to bacterial infection. Many studies have shown that IL-10 influences the homeostasis of the intestinal mucosa via regulation of several signaling pathways. IL10 regulates the expression and activities of pro-inflammatory cytokines via growth inhibition and differentiation-related signaling, such as that involving the phosphatidylinositol-4,5- 
A
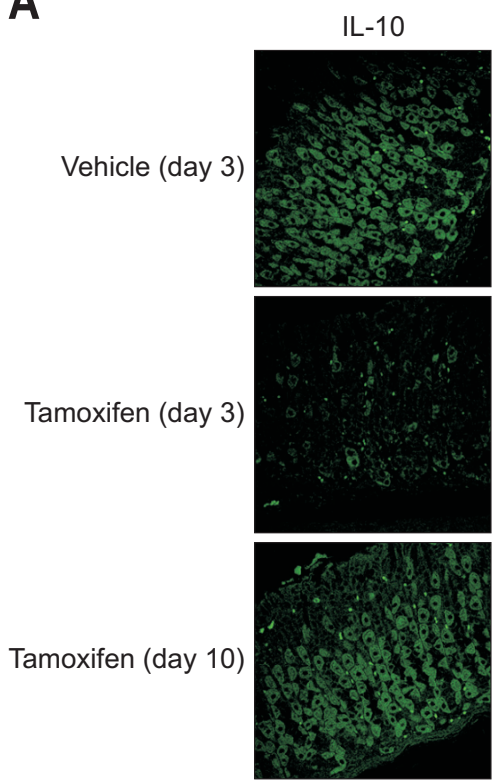

Tamoxifen (day 21)

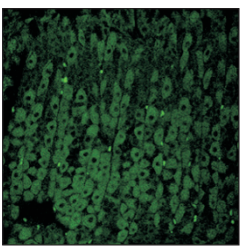

B

Vehicle (day 3)

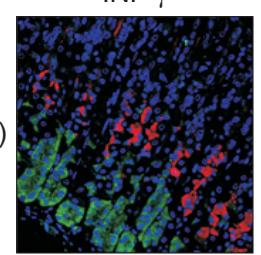

Tamoxifen (day 3)
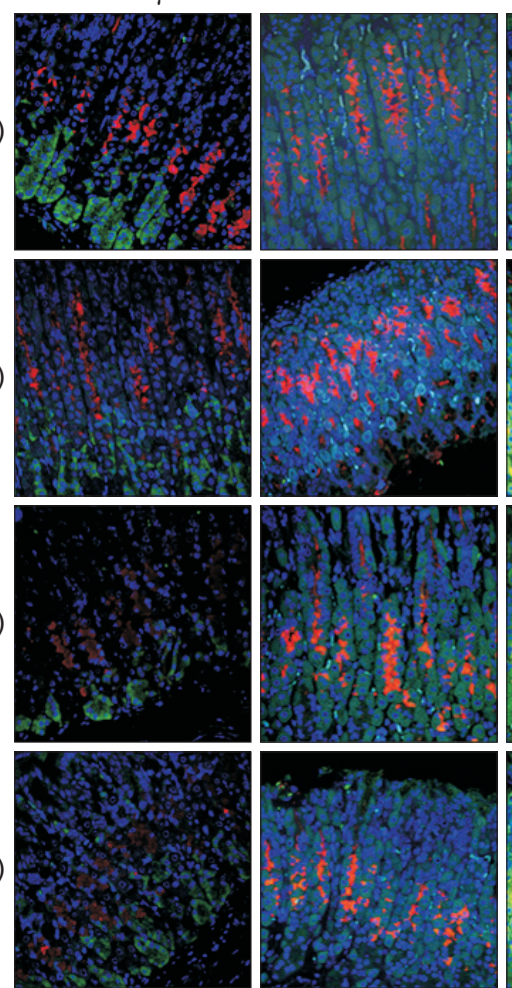
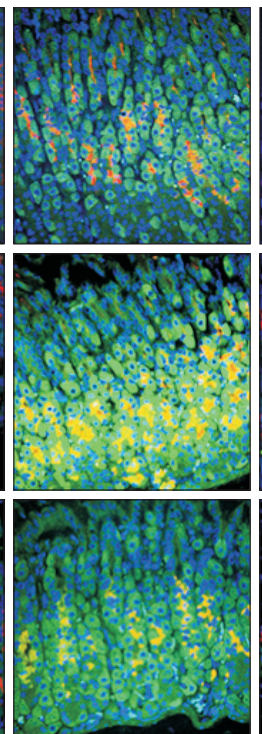

IL-10+VEGF- $\beta$
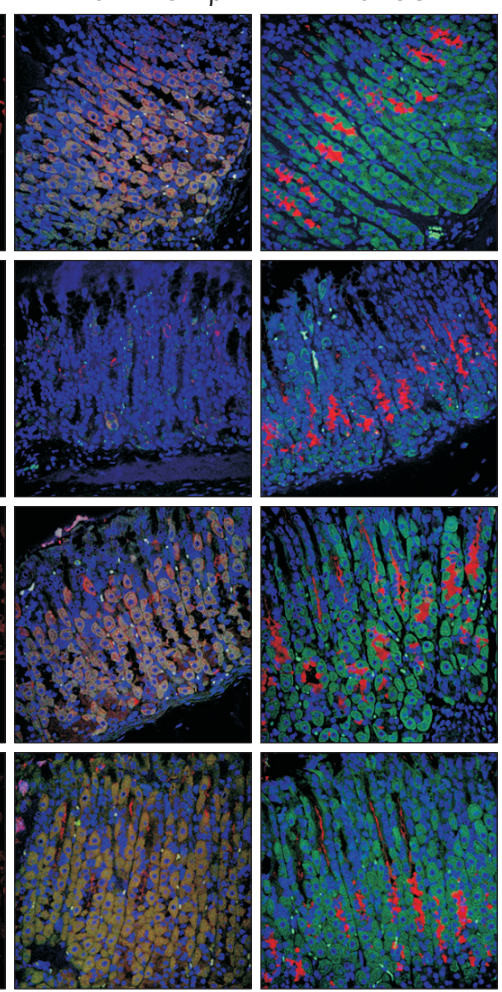

$\mathrm{IL}-1 \beta$
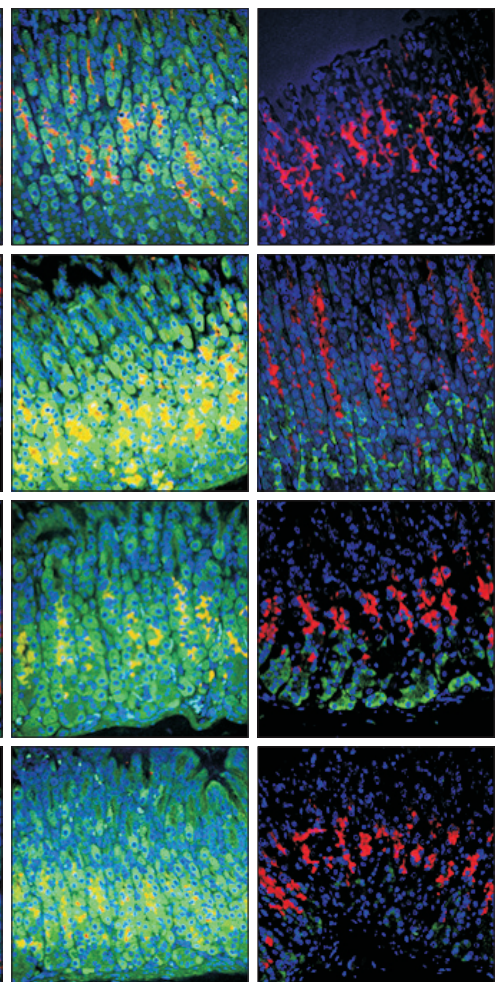

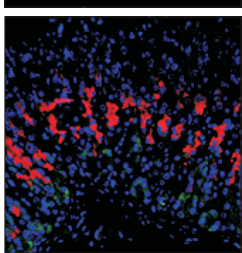

Fig. 4. Interleukin (IL)-10 expression is associated with parietal cells (PCs). (A) The expression level of vascular endothelial growth factor (VEGF)- $\beta$ and IL-10 were evaluated by confocal microscopy after dual immunofluorescent staining of deparaffinized tissue sections (red: GS II, a mucosal neck cell marker; green: VEGF- $\beta$, a PC marker). (B) The expression levels of interferon (IFN)- $\gamma$, IL-6, tumor necrosis factor (TNF)- $\alpha$, and IL- $1 \beta$ were evaluated by confocal microscopy after dual immunofluorescence staining of deparaffinized tissue sections (red: GS II, a mucosal neck cell marker; green: each cytokine). bisphosphate 3-kinase pathway, the Janus kinase/signal transducer and activator of transcription pathway, and the mitogenactivated protein kinase pathway in the intestinal mucosa. ${ }^{13,22}$
Recent studies have also suggested that genetic variations in IL-10 are associated with inflammatory responses and atrophy in gastric tissues. ${ }^{23}$ In our study, the expression level of IL- 
10 showed the same tendency toward changes in PCs, and IL10 -secreting cells co-localized with the PC marker VEGF- $\beta$ (Fig. 3). Since the loss of PCs can initiate SPEM, IL-10 reduction induced by PC loss was closely associated with the development of SPEM in tamoxifen-treated mice. The role of IL-10 in cancer has become less certain with the greater understanding of its immune-stimulatory activity. ${ }^{24}$ Although IL-10 is needed for Thelper cell function, T-cell immune surveillance, and suppression of cancer-associated inflammation, it may also be a key cytokine in the host's battle against cancer during early gastric carcinogenesis.

Consequently, we evaluated the change of cytokine expression during the tamoxifen-induced SPEM and identified that IL-10 expression was markedly decreased at the time of SPEM development and subsequently returned to normal, accompanied by a reversal in histologic changes. IL-10 may regulate the homeostasis of the gastric mucosa and inhibit the development of mucosal metaplasia, and it may play a potential therapeutic role in inhibiting the development of SPEM during early gastric carcinogenesis. Future studies must investigate the role of epithelial IL-10 in order to elucidate the mechanism of SPEM initiation in gastric tissue.

\section{CONFLICTS OF INTEREST}

No potential conflict of interest relevant to this article was reported.

\section{ACKNOWLEDGEMENTS}

This research was supported by the Basic Science Research Program through the National Research Foundation of Korea (NRF) funded by the Ministry of Education, Science and Technology (2012R1A1A1005646) and by a grant from SK Chemical Research Fund of the Korean Society of Gastroenterology in 2014.

\section{REFERENCES}

1. Bordi C, D’Adda T, Baggi MT, Pilato FP. Structure and function of endocrine cells in the oxyntic (acid-secreting) mucosa of human stomach. Scand J Gastroenterol Suppl 1989;166:115-121.

2. Goldenring JR, Nam KT. Oxyntic atrophy, metaplasia, and gastric cancer. Prog Mol Biol Transl Sci 2010;96:117-131.

3. Weis VG, Goldenring JR. Current understanding of SPEM and its standing in the preneoplastic process. Gastric Cancer 2009;12:189197.

4. Fox JG, Wang TC. Inflammation, atrophy, and gastric cancer. J Clin Invest 2007;117:60-69.

5. Nozaki K, Ogawa M, Williams JA, et al. A molecular signature of gastric metaplasia arising in response to acute parietal cell loss. Gastroenterology 2008;134:511-522.
6. Petersen CP, Weis VG, Nam KT, Sousa JF, Fingleton B, Goldenring JR. Macrophages promote progression of spasmolytic polypeptideexpressing metaplasia after acute loss of parietal cells. Gastroenterology 2014;146:1727-1738.e8.

7. Nam KT, O’Neal RL, Coffey RJ, Finke PE, Barker N, Goldenring JR. Spasmolytic polypeptide-expressing metaplasia (SPEM) in the gastric oxyntic mucosa does not arise from Lgr5-expressing cells. Gut 2012;61:1678-1685.

8. Abdiev S, Ahn KS, Khadjibaev A, et al. Helicobacter pylori infection and cytokine gene polymorphisms in Uzbeks. Nagoya J Med Sci 2010;72:167-172.

9. Sugimoto M, Yamaoka Y, Furuta T. Influence of interleukin polymorphisms on development of gastric cancer and peptic ulcer. World J Gastroenterol 2010;16:1188-1200.

10. Jung HC, Kim JM, Song IS, Kim CY. Helicobacter pylori induces an array of pro-inflammatory cytokines in human gastric epithelial cells: quantification of mRNA for interleukin-8, -1 alpha/beta, granulocyte-macrophage colony-stimulating factor, monocyte chemoattractant protein-1 and tumour necrosis factor-alpha. J Gastroenterol Hepatol 1997;12:473-480.

11. Ramis IB, Vianna JS, Gonçalves CV, von Groll A, Dellagostin OA, da Silva PEA. Polymorphisms of the IL-6, IL-8 and IL-10 genes and the risk of gastric pathology in patients infected with Helicobacter pylori. J Microbiol Immunol Infect 2017;50:153-159.

12. Konturek PC, Kania J, Konturek JW, Nikiforuk A, Konturek SJ, Hahn EG. H. pylori infection, atrophic gastritis, cytokines, gastrin, COX-2, PPAR gamma and impaired apoptosis in gastric carcinogenesis. Med Sci Monit 2003;9:SR53-SR66.

13. Howlett M, Chalinor HV, Buzzelli JN, et al. IL-11 is a parietal cell cytokine that induces atrophic gastritis. Gut 2012;61:1398-1409.

14. Ogawa M, Nomura S, Car BD, Goldenring JR. Omeprazole treatment ameliorates oxyntic atrophy induced by DMP-777. Dig Dis Sci 2006;51:431-439.

15. Weis VG, Sousa JF, LaFleur BJ, et al. Heterogeneity in mouse spasmolytic polypeptide-expressing metaplasia lineages identifies markers of metaplastic progression. Gut 2013;62:1270-1279.

16. Lefebvre 0, Chenard MP, Masson R, et al. Gastric mucosa abnormalities and tumorigenesis in mice lacking the pS2 trefoil protein. Science 1996;274:259-262.

17. Huh WJ, Khurana SS, Geahlen JH, Kohli K, Waller RA, Mills JC. Tamoxifen induces rapid, reversible atrophy, and metaplasia in mouse stomach. Gastroenterology 2012;142:21-24.e7.

18. Chandanos E, Lindblad M, Jia C, Rubio CA, Ye W, Lagergren J. Tamoxifen exposure and risk of oesophageal and gastric adenocarcinoma: a population-based cohort study of breast cancer patients in Sweden. Br J Cancer 2006;95:118-122.

19. Polin SA, Ascher SM. The effect of tamoxifen on the genital tract. Cancer Imaging 2008;8:135-145.

20. Giraud AS, Jackson C, Menheniott TR, Judd LM. Differentiation of the gastric mucosa IV: role of trefoil peptides and IL-6 cytokine family signaling in gastric homeostasis. Am J Physiol Gastrointest Liver Physiol 2007;292:G1-G5. 
21. De Vita F, Orditura M, Galizia G, et al. Serum interleukin-10 levels in patients with advanced gastrointestinal malignancies. Cancer 1999;86:1936-1943.

22. Yamaoka Y, Yamauchi K, Ota H, et al. Natural history of gastric mucosal cytokine expression in Helicobacter pylori gastritis in Mongolian gerbils. Infect Immun 2005;73:2205-2212.

23. Bodger K, Wyatt JI, Heatley RV. Gastric mucosal secretion of in- terleukin-10: relations to histopathology, Helicobacter pylori status, and tumour necrosis factor-alpha secretion. Gut 1997;40:739744.

24. Dennis KL, Blatner NR, Gounari F, Khazaie K. Current status of interleukin-10 and regulatory T-cells in cancer. Curr Opin Oncol 2013;25:637-645. 